\title{
Surgical Role in Management of Intracranial Germ Cell Tumors in Pediatric Age Group
}

\author{
Mohamed Reda ${ }^{1,}$, , Mohamed Elbeltagy ${ }^{1}$, Mohamed Kamal ${ }^{2}$, Mahmoud Hammad $^{3}$ \\ ${ }^{1}$ Department of Neurosurgery, Kasralainy Medical School, Cairo University and Children's Cancer Hospital Egypt, Cairo, Egypt \\ ${ }^{2}$ Department of Research Children's Cancer Hospital Egypt, Cairo, Egypt \\ ${ }^{3}$ Department of Pediatric Oncology, National Cancer Institute, Cairo University and Children's Cancer Hospital Egypt, Cairo, Egypt
}

Email address:

Mohamed.redaa@57357.org (M. Reda)

*Corresponding author

\section{To cite this article:}

Mohamed Reda, Mohamed Elbeltagy, Mohamed Kamal, Mahmoud Hammad. Surgical Role in Management of Intracranial Germ Cell Tumors in Pediatric Age Group. Cancer Research Journal. Vol. 7, No. 2, 2019, pp. 66-72. doi: 10.11648/j.crj.20190702.16

Received: May 14, 2019; Accepted: June 12, 2019; Published: June 24, 2019

\begin{abstract}
Introduction: Primary intracranial germ cell tumors (ICGCTs) are rare, histologically diverse, and diagnostically challenging tumors that are usually localized in the pineal and suprasellar regions of the brain. Advanced neurosurgical techniques such as neuroendoscopy and frameless stereotactic biopsy have made diagnosis of newly discovered cases of ICGCTs easier and safer. Material and methods: Seventeen patients with intracranial germ cell tumors operated upon between 2008 to 2012 at the Children's Cancer Hospital Egypt, were retrospectively reviewed and analyzed regarding the surgical decision, clinical outcome and surgical complications. Results: There were 9 cases of germinoma (53\%), and 8 cases of nongerminomatous germ cell tumors (47\%). Nine cases were in the pineal region, six in the suprasellar, and two in the thalamic region. Ten cases were operated upon initially by open surgery and frozen section with subtotal resection and seven cases were biopsied either endoscopically ( 3 cases) or by frameless guided stereotaxic (4 cases). Accurate pathology was achieved in all biopsied cases without major complications. In the germinoma group, the 4-year overall survival and progression free survival rate were $75 \%$ for both at a median follow up period of 26 (range $1-50$ ) months. For the non-germinomatous germ cell tumors group, the 4-year OS and PFS rates were $36.5 \%$ and $31.2 \%$ at a median follow up period of 11 (range 2-54) months, respectively. Conclusion: In cases of intracranial germ cell tumors with negative tumor markers the role of surgery is important in the establishment of proper histopathological diagnosis. However, in Non Germinomatous Germ Cell Tumors, further investigations should be done regarding the extent of resection owing to the poor long-term outcome.
\end{abstract}

Keywords: Intracranial Germ Cell Tumors, Pediatric, Endoscopic Biopsy, Stereotactic Biopsy

\section{Introduction}

The central nervous system (CNS) is a common site for extragonadal germ cell tumors (GCTs). Primary intracranial germ cell tumors are rare malignancies and diagnostically challenging with wide pathological diversity [1]. Intracranial Germ cell tumors (ICGCTs) account approximately for 3-5\% of brain tumors with a peak incidence from 10 to 19 years old. They are usually localized in midline structures and nearly half of ICGCTs are located in the pineal and suprasellar region of the brain [2-3]. Other CNS sites are less frequently involved. There is an obvious male predominance; however, this finding is more associated with pineal region tumors [4].
ICGCTs are classified into two histological categories: germinomatous and nongerminomatous germ cell (NGGCT). The NGGCTs are subclassified histologically into yolk sac tumor (YST), embryonal carcinoma (EC), choriocarcinoma (CC), teratoma and more commonly mixed germ cell tumor [5].

The histopathology of GCTs has prognostic significance and are subclassified into good prognosis (pure germinoma and mature teratoma), intermediate prognosis group (germinoma with elevated levels of $\mathrm{B}-\mathrm{HCG}$, immature teratoma, teratoma with malignant transformation, and mixed tumors mainly consisting of germinoma or teratomas) and poor prognosis group (yolk sac tumor, embryonal carcinoma, choriocarcinoma, and mixed tumors consisting mainly of these histological subtypes) [6]. 
Neurosurgical intervention is indicated for diagnosis in the absence of elevated tumor markers, CSF diversion for relieving associated hydrocephalus, and tumor debulking or second look surgery for cases without adequate response after neoadjuvant therapy. Advanced neurosurgical techniques such as neuroendoscopy and frameless stereotactic biopsy have made the diagnosis of ICGCTs easier and safer. Microsurgical techniques and navigation system even made conventional surgery easier with less associated morbidities [7].

Pure germinomas are highly radiosensitive tumors with more than $80 \%$ five-year survival rates with radiotherapy alone [8-9]. Combined chemotherapy and reduced radiation volume and dose have increasingly been utilized especially in children prior to puberty. Same survival rates have been observed with such combination therapies. Clinical trials changing from involved field radiation (IFR) to whole ventricular irradiation (WVI) following chemotherapy for non-metastatic CNS germinoma resulted in a local control rate over $90 \%[3,10]$. Trials for chemotherapy alone and omitting radiotherapy resulted in inferior survival rates of $68 \%$ [11].

The standard treatment of NGGCT is neoadjuvant chemotherapy followed by craniospinal irradiation (CSI) given at a dose of 30-36 Gy with a boost to the primary tumor site for a total of 54-60 Gy which resulted in a survival rate of $60-70 \%$. Salvage and a second look surgery for residual disease after chemo-radiotherapy is highly encouraged in cases without complete remission [11-13].

Herein, we describe the surgical results using different techniques to diagnose pediatric ICGCTs treated at Children's Cancer Hospital Egypt.

\section{Patients and Methods}

Retrospective data analysis was done for seventeen patients with intracranial germ cell tumors operated upon between 2008 - 2012 at the Children's Cancer Hospital Egypt. Data was partially presented in the $15^{\text {th }}$ world conference of neurosurgery (WFNS).

All patients were new cases and below 18 years of age at presentation. Initial disease characteristics were collected and included; age, histopathology, primary tumor location, magnetic resonance imaging (MRI) of brain and spine, alphafetoprotein (AFP) and beta human chorionic gonadotropin (beta-HCG) in both serum and cerebrospinal fluid (CSF), description of surgical procedures and its complications, and the survival outcome.

\subsection{Surgical Strategy (Differs According to the Site of the GCT and the Presence of Hydrocephalus)}

\subsubsection{Pineal Region GCT}

Serum tumor markers is obtained at time of presentation and CSF tumor markers is taken from all cases either at the time of CSF diversion and endoscopic biopsy, if the patient is hydrocephalic, or through lumbar puncture.

Simultaneous endoscopic third ventriculostomy (ETV) and endoscopic biopsy (EB) were performed for pineal region tumors extending to the posterior part of the third ventricle with obstructive hydrocephalus; both procedures were done through the same burr hole, $2 \mathrm{~cm}$ in front of the coronal suture $3 \mathrm{~cm}$ from the midline. Zero angle diagnostic rigid endoscope was introduced at first to check the anatomy of the third ventricle and to exclude any dissemination of the tumor and the tumor surface. Then the working channel was entered to perform a third ventriculostomy. Three biopsies should be taken from different sites using endoscopic biopsy forceps. It is important to keep on continuous irrigation to control bleeding. Monopolar cauterization was rarely needed at the site of the biopsies.

Frameless stereotactic biopsy was performed for pineal region tumors in absence of ventriculomegaly. Frameless Stereotactic Navigation system Vario Guide was used. Preoperative MRI was uploaded to the brain lab followed by marking the target and choosing the trajectory. Patient registration is performed using Z-touch technique. Then we targeted Pre-planned trajectory with vario guide. Biopsy needle $1.8 \mathrm{~mm}$ is used to take the biopsy.

Open resection was usually done for all cases, before changing the protocol to endoscopic or stereotactic biopsy. Now open resection is only reserved for cases that can benefit from second look surgery in cases of NGGCT with no remission after adjuvant therapy. Many approaches were described but infratentorial supracerebellar approach in a sitting position was our preference.

\subsubsection{Thalamic Region GCT}

Open resection was used to be done for all thalamic region tumors but replaced by frameless stereotactic biopsy. If there is a hydrocephalus, ventriculoperitoneal (VP) shunt was introduced and CSF sample for tumor markers was withdrawn. In case of positive tumor markers, no further surgical interference was done but if proven to be negative a stereotactic biopsy was performed. Open surgery was reserved only for cases who will benefit from a second look surgery in cases of NGGCT with progression or incomplete response after chemotherapy.

\subsubsection{Sellar and Suprasellar Region GCT}

If there is hydrocephalus, we perform (VP) shunt and take a CSF sample for tumor markers, if positive no further surgical interference will be needed, but if negative we shift to an open resection with intraoperative frozen section pathology if proven to be GCT, trails for a gross total resection is not mandatory.

\subsection{Treatment Plan}

\subsubsection{Germinoma}

Initial evaluation with MRI brain/spine to diagnose and assess the disease extent. AFP and beta-HCG in both serum and cerebrospinal fluid (CSF) were done in all patients. Whole ventricular irradiation (WVI) were given at a dose of 25 to 30 Gy with 15 Gy boost to the primary site reaching a total dose of 40 to $45 \mathrm{~Gy}$ (with 1.5 - to $2 \mathrm{~cm}$ margin). In case 
of leptomeningeal dissemination, craniospinal irradiation was given at a dose of 30 to 35 Gy with boost a to the primary site to reach 40 to $45 \mathrm{~Gy}$.

\subsubsection{Nongerminomatous GCT Treatment Protocol}

Our center follows the Children's Oncology Group (COG) ACNS0122 trial for NGGCT patients, which uses neoadjuvant combination of carboplatin/etoposide (Cycles 1, 3 , and 5) alternating with ifosfamide/etoposide (Cycles 2, 4, and 6) for a total of 6 induction cycles. The duration of the induction period is approximately 18 weeks unless delay occurs due to myelosuppression or other toxicities. Response is evaluated by MRI brain/spine and tumor markers after each 3 cycles. After end of induction patient is evaluated for either the start or radiotherapy or a second look surgery and irradiation thereafter (Figure 1). The protocol details and doses are mentioned elsewhere under ClinicalTrials.gov Identifier NCT00047320.
Patients in complete remission (CR) after induction will receive craniospinal irradiation CSI at 36 Gy with tumor bed involved field boost to reach $54 \mathrm{~Gy}$ in $1.8 \mathrm{~Gy}$ daily fractions. Other patients with less than CR, a second- look surgery is strongly recommended. Patients who achieve $\mathrm{CR} / \mathrm{PR}$ via second-look surgery will then proceed to radiation therapy as previously described. In patients whom a second-look surgery is not possible, will proceed to radiotherapy hoping for further tumor regression.

\subsection{Statistical Methods}

Progression-free and overall survival rates were defined as months to tumor progression or death from any cause, and months to death from any cause or last follow-up, respectively. The Kaplan-Meier method was used to estimate the survival probabilities of PFS and OS.

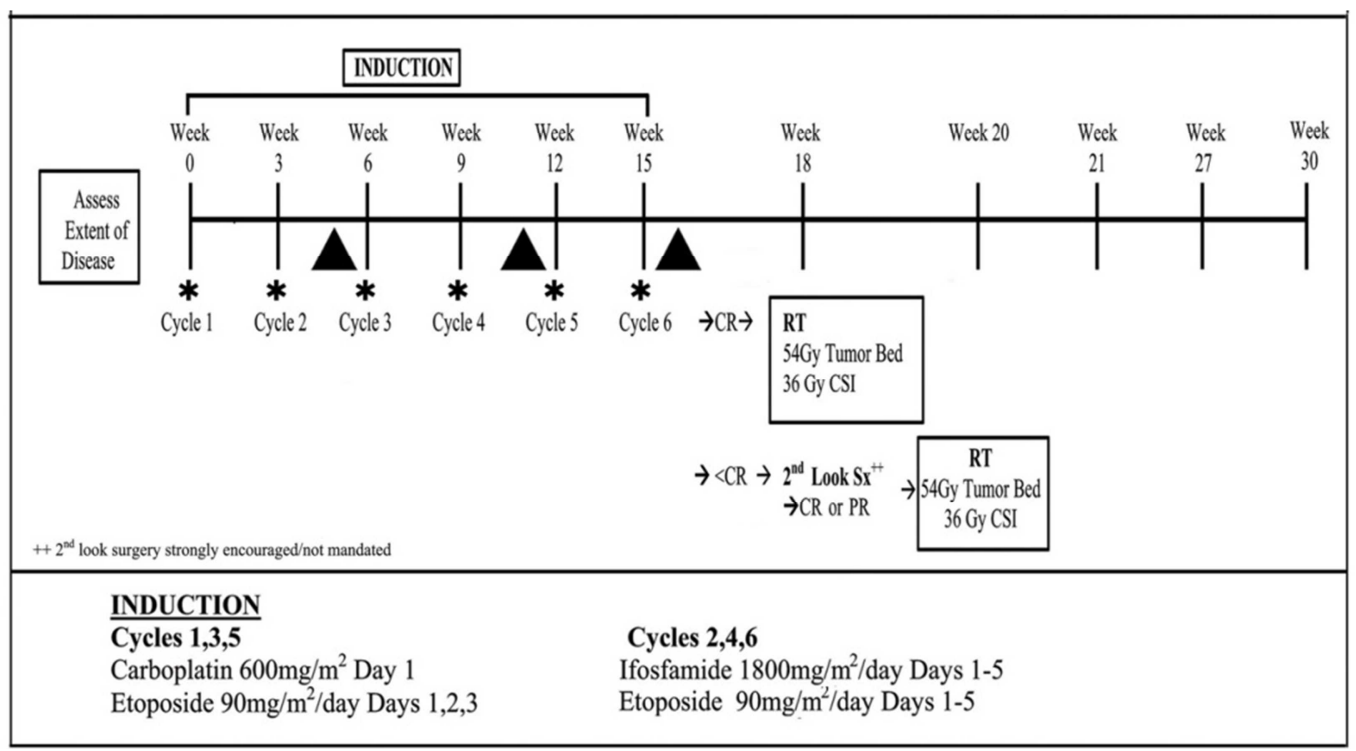

RT: radiotherapy, * at the beginning of each cycle, absolute neutrophilic count $>750$ and platelets $>75, \boldsymbol{M R I}$ scan

Figure 1. Treatment protocol for non germinomatous germ cell tumor (modified and adopted from COG ACS0122: NCT00047320).

\section{Results}

Patient and disease characteristics are summarized in table 1. Median age at diagnosis was 9 years (range: 2-18 years). Male predominance was observed $(64.7 \%)$, as well as pineal region GCTs (53.0\%). The suprasellar and thalamic regions were observed in six patients $(35.3 \%)$ and two cases $(11.7 \%)$, respectively.

Table 1. Patient demographic data and disease characteristics.

\begin{tabular}{lll}
\hline Variable & Number of patients & Percentage (\%) \\
\hline Total number of patients & 17 & $100 \%$ \\
Age in years (median; range) & 9 years $(2-18)$ & \\
Gender & & \\
Male & 11 & $64.7 \%$ \\
Female & 6 & $35.2 \%$ \\
Tumor pathology & & \\
Germinoma & 9 & $53.0 \%$ \\
NGGCT & & \\
\hline
\end{tabular}

\begin{tabular}{lll}
\hline Variable & Number of patients & Percentage (\%) \\
\hline Malignant mixed GCT & 5 & $29.4 \%$ \\
Immature teratoma & 2 & $11.7 \%$ \\
Yolk Sac tumor & 1 & $5.88 \%$ \\
Tumor Location & & \\
Pineal & 9 & $53.0 \%$ \\
Suprasellar & 6 & $35.3 \%$ \\
Thalamic & 2 & $11.7 \%$ \\
Type of surgical procedure & & \\
Open resection & 10 & $58.8 \%$ \\
Endoscopic biopsy & 3 & $17.6 \%$ \\
Stereotactic biopsy & 4 & $23.5 \%$ \\
\hline
\end{tabular}

Histopathology: Almost half of patients were pure germinoma $(n=9)$; all of them had normal serum and CSF tumor markers. Eight patients were NGGCT and according to Matsutani et al classification [6], were categorized as follows: poor prognosis group in 6 patients (mixed germ cell tumors; $29.4 \%$, and yolk sac tumor; $5.9 \%$ ) and intermediate risk group in 2 patients with immature teratoma (11.7\%). Tumor 
markers in NGGCT were high only in 3 patients; two with elevated AFP and one with high beta HCG (embryonal carcinoma component in the mixed GCT), while other patients were diagnosed only after surgical intervention and pathological confirmation.

Surgical results:

Pineal region GCT

Endoscopic biopsy at time of ventriculostomy was performed in three patients, histopathology was informative in all cases and was proved to be germinoma in two patients and NGGCT in 1 patient (Figure 2). No major complications occurred from the procedure, however one patient developed transient left sided weakness post-operative and improved within 2 weeks.

Frameless Stereotactic navigated biopsy was performed in three patients, all of them were germinoma by histopathology with no complications occurred from the procedure.

Open resection was performed in three patients through supracerebellar infratentorial approach with total resection in one case and subtotal resection in the other two patients. Frozen section pathology was performed intraoperatively and proved to be germ cell tumors in the three patients. The final histopathology was germinoma in two patients and NGCST in another one. No post-operative morbidity or mortality occurred in any of these cases.

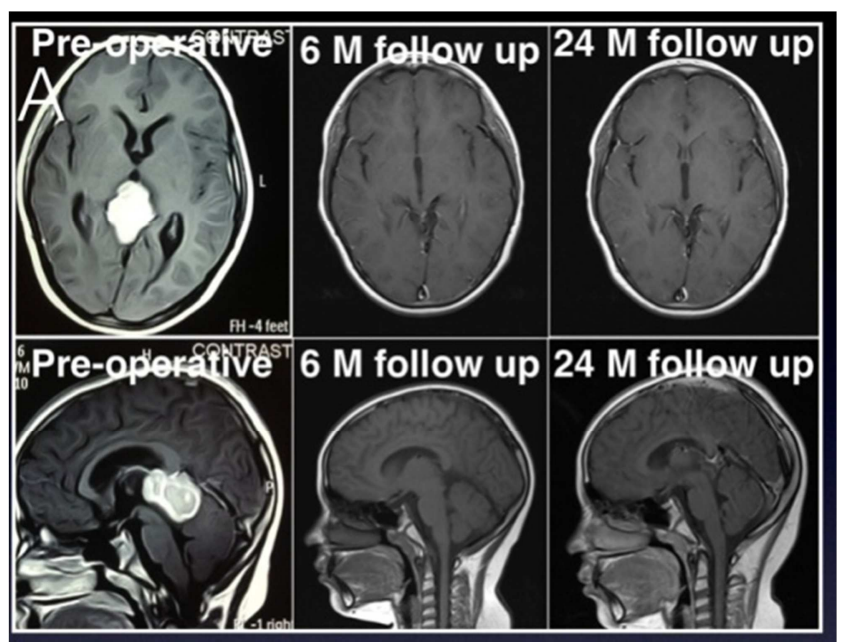

Figure 2. Endoscopic biopsy for eight years old male child diagnosed with pure germinoma. Pre-operative images, and follow up after 6- and 24months post radiation therapy that shows a complete remission in follow up period.

\section{Suprasellar GCT}

Six cases with suprasellar Germ cell tumors were operated by open resection through pterional transsylvian approach with microsurgical techniques; intraoperative frozen section was done and once germ cell tumor was suspected, the aim of the surgery was changed to safe debulking. Final pathology was germinoma in two cases and NGGCT in the other four cases. No surgical complications were reported from the open resection procedure.

Thalamic GCT

Open resection with microsurgical techniques was done in one case, and another case was operated by frameless stereotactic biopsy. The pathology was NGGCT in both cases. Second look surgery was done for one of the NGGCT after inadequate response to neoadjuvant therapy.

Disease Outcome: In the germinoma group, the 4-year overall survival (OS) and progression free survival rate (PFS) were $75 \%$ for both at a median follow up period of 26 (range 10-50) months. For the nongerminomatous germ cell tumors group, the 4-year overall and progression free survival rates were $36.5 \%$ and $31.2 \%$, respectively at a median follow up period of 11 (range 2-54) months (Figure 3 and 4). There was a significant difference in the PFS $(\mathrm{p}<0.05)$ between germinoma and NGGCT groups. However, the difference in the OS between the two groups was not significant $(p>0.05)$.

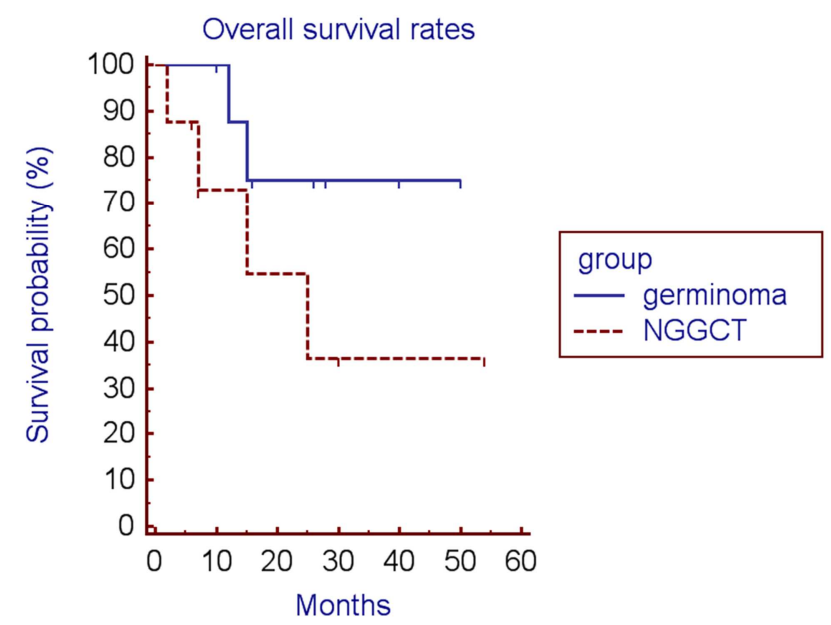

Figure 3. The four-year overall survival rates for GCTs cases.

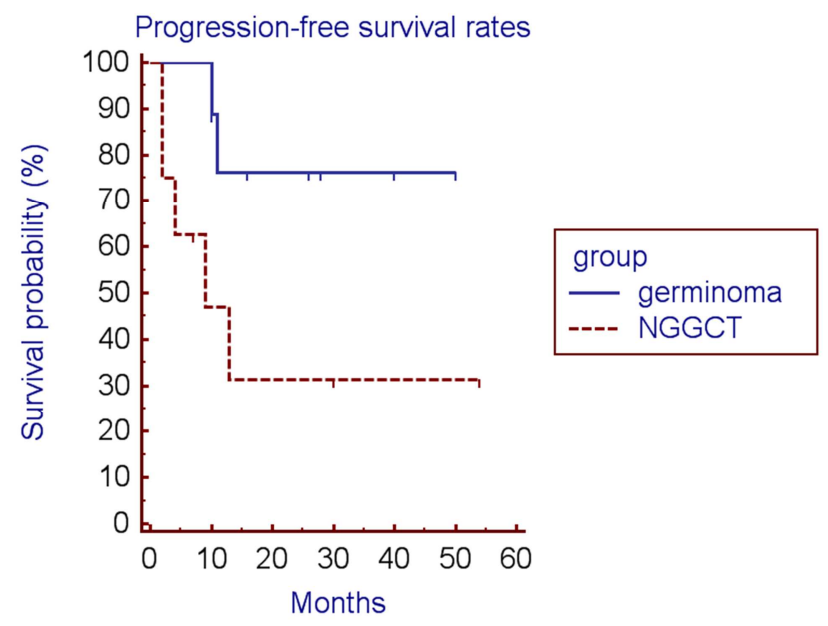

Figure 4. The four-year progression free survival rates for GCTs cases.

\section{Discussion}

Intracranial GCTs are morphologically diverse group of neoplasms. They are relatively rare with a peak incidence at 11 years of age and approximately $90 \%$ of GCTs occur in patients under 20 years old [14]. Male predominance is described in literature, especially for pineal region lesions [15], that was in consistence with our results, in which 8 out 
of 9 pineal region GCTs tumors were males. Five histologically diverse subtypes are described; germinomas and NGGCTs that include (ECs, YST, teratoma and CC) [5]. Approximately $25 \%$ of intracranial GCTs are mixed subtypes with the outcome related to the treatment of the most malignant component [16]. Treatment options include, open surgery for resection of the tumor, radio- and chemo- therapy, and CSF diversion which include the use of VP shunts, external ventricular drains (EVD) or ETVs. Most of the time emergency situations dictates the rapid intervention for lifethreatening hydrocephalus [17].

Surgical biopsy is needed for the diagnosis of ICGCTs and histological interpretation, except in those cases with characteristic elevations of tumor markers. In case of borderline elevation above the normal range, a surgical biopsy is strongly mandated. Biopsies have some limitations related to the tumor location and the associated morbidities, as well as sampling errors in case of small tissue samples [18]. In the current study, histological samples interpretation was done in $100 \%$ of cases with no major complications compared to others who reported a variable success rate from $69 \%$ to $100 \%$ [19-21].

The extent of surgical resection in ICGCT is a matter of debate; still the role for partial or gross total resection in germinomas is not indicated being a highly radiosensitive tumors, and radical resection in NGGCTs is still unclear, except in cases that do not achieve complete response after chemotherapy, a second-look surgery should be considered, especially in cases that have normalization of tumor markers $[4,22]$. In this study, seventeen cases of ICGCTs were operated including 9 cases of pure germinoma with negative tumor markers including seven cases located in the pineal region and the other 8 cases were NGGCTs. Studies described similarly that, germinomas account for approximately $50 \%-70 \%$ of cases and the remaining third comprises the NGGCTs [16, 23].

We operated 3 pineal region lesions by endoscopic biopsy and ETV at same time of the procedure. Anatomical feature of the ventricle makes it a safe and easy for using the rigid endoscope with a single burr hole technique, good illumination with continuous irrigation allows us to safely choose the target place of the biopsy and also examine the ependymal wall for any dissemination. ETV is now preferred by many neurosurgeons than VP shunts as an initial approach to cases with obstructive hydrocephalus with a mean 2 years follow up with $70 \%$ success rate [24]. Others described either single trajectory approach performing both biopsy and ventriculostomy through same burr hole usually precoronal about $3 \mathrm{~cm}$ from the midline $[20,25,26]$ or double burr hole technique coronal for ETV and frontal for endoscopic biopsy (EB) [20-21]. Another 10 cases in this study were operated by open resection with total or subtotal resection achieved, the tumor location was suprasellar, pineal and thalamic region in 6,3 and one patients, respectively. Frozen section is indicated to define the pathology and if germ cell tumor is confirmed, the aim of surgery is switched to debulking rather than an aggressive radical surgery. Frameless stereotactic biopsy is a safe procedure with high diagnostic yield about $93.8 \%$ to $99 \%$, with low incidence of morbidities, and hemorrhagic complications reported from this procedure ranges from zero to $7 \%$ [27-29]. In this study stereotactic biopsy was performed safely in 4 patients, three with pineal region tumors and one with thalamic tumor, with the confirmation of histopathology in all of them.

Since germinoma is highly radiosensitive and completely melts with radiation alone [14, 30], so safe surgery is mandated only to confirm the diagnosis and the rapid start of radiation therapy thereafter. At 4 years, the survival rates of this study for germinoma group were $75 \%$ for both OS and PFS with median follow up period of 26 month. Similarly, others studies reported, 5-year OS rate of $80 \%[8,9,12]$. Better survival rates have been reported using radiation alone with a 5-year survival rates $>90 \%$; however, the effective radiotherapy dose and field remain controversial [6, 31]. The role of chemotherapy followed by reduced dose of radiation was discussed to avoid late complications especially in patients prior to puberty $[3,12]$.

In the NGGCT group of patients in this study, all patients received postoperative chemotherapy and CSI. A second look surgery was needed in one case which didn't show adequate response to adjuvant therapy. Many international studies have now included second look surgery in their treatment protocols for ICGCTs [4].

In this study, the 4-year OS and PFS rates were $36.5 \%$ and $31.2 \%$ at a median follow up period of 11 (range 2-54) months, respectively. Others described better survival rates with a 4-year EFS and OS rates of $67 \%$ and $74 \%$, respectively with chemotherapy followed by CSI with or without second look surgery [32-34]. An explanation to the modest response described in this study could be related to the small number of included patients, as well as the poor histological criteria of patients included in the NGCCT group (mixed germ cell tumors; 5 cases, and yolk sac tumor; 1 case). Kanomori et al reported the 3 -year survival rates for 8 patients with poor histological criteria as $56 \%$ for OS and $29 \%$ for PFS [12].

\section{Conclusion}

The role of surgical biopsy in intracranial germ cell tumors with negative tumor markers is important to establish a proper histopathological diagnosis; however, small group of cases can be diagnosed based on their tumor markers. Histological subtyping is important to define the plan of treatment which reflects on the chemotherapy used, the field and dose of radiotherapy given, as well as the extent of surgical resection. Endoscopic third ventriculostomy (ETV) and endoscopic biopsy or frameless stereotactic biopsy are safe surgical techniques to obtain adequate biopsies compared to open resection. More collaborative efforts and new treatment modalities are still needed to improve the survival outcome especially in the NGGCTs group, which show less sensitivity to radiotherapy than germinoma group and to reach a reduced radiotherapy dose to avoid late effects 
and morbidities.

\section{References}

[1] Villano JL, Virk IY, Ramirez V, Propp JM, Engelhard HH, McCarthy BJ. Descriptive epidemiology of central nervous system germ cell tumors: nonpineal analysis. Neuro Oncol, 2010 12: 257-264.

[2] Dolecek TA, Propp JM, Stroup NE, Kruchko C. CBTRUS Statistical Report: Primary Brain and Central Nervous System Tumors Diagnosed in the United States in 2005-2009. Neuro Oncol, 2012 14: v1-v49.

[3] Matsutani M, Japanese Pediatric Brain Tumor Study Group. Combined chemotherapy and radiation therapy for CNS germ cell tumors--the Japanese experience. J Neurooncol, 2001 54: 311-6.

[4] Echevarria ME, Fangusaro J, Goldman S. Pediatric Central Nervous System Germ Cell Tumors: A Review. Oncologist, 2008 13: 690-699.

[5] Fetcko K, Dey M. Primary Central Nervous System Germ Cell Tumors: A Review and Update HHS Public Access. Med Res Arch, 2018

[6] Matsutani M, Sano K, Takakura K, Fujimaki T, Nakamura O, Funata N, Seto T. Primary intracranial germ cell tumors: a clinical analysis of 153 histologically verified cases. J Neurosurg, 1997 86: 446-455.

[7] Souweidane MM, Krieger MD, Weiner HL, Finlay JL. Surgical management of primary central nervous system germ cell tumors. J Neurosurg Pediatr, 2010 6: 125-130.

[8] Linstadt D, Wara WM, Edwards MSB, Hudgins RJ, Sheline GE. Radiotherapy of primary intracranial germinomas: The case against routine craniospinal irradiation. Int $\mathrm{J}$ Radiat Oncol Biol Phys, 1988 15: 291-297.

[9] Shirato H, Nishio M, Sawamura Y, Myohjin M, Kitahara T, Nishioka T, Mizutani Y, Abe H, Miyasaka K. Analysis of longterm treatment of intracranial germinoma. Int $\mathrm{J}$ Radiat Oncol Biol Phys, 1997 37: 511-515.

[10] Calaminus G, Kortmann R, Worch J, Nicholson JC, Alapetite C, Garrè ML, Patte C, Ricardi U, Saran F, Frappaz D. SIOP CNS GCT 96: Final report of outcome of a prospective, multinational nonrandomized trial for children and adults with intracranial germinoma, comparing craniospinal irradiation alone with chemotherapy followed by focal primary site irradiation for patients with localized disease. Neuro Oncol, 2013 15: 788-796.

[11] Kellie SJ, Boyce H, Dunkel IJ, Diez B, Rosenblum M, Brualdi L, Finlay JL. Intensive cisplatin and cyclophosphamide-based chemotherapy without radiotherapy for intracranial germinomas: Failure of a primary chemotherapy approach. Pediatr Blood Cancer, 2004 43: 126-133.

[12] Kanamori M, Kumabe T, Saito R, Yamashita Y, Sonoda Y, Ariga H, Takai Y, Tominaga T. Optimal treatment strategy for intracranial germ cell tumors: a single institution analysis. J Neurosurg Pediatr, 2009 4: 506-514.

[13] Weiner HL, Lichtenbaum RA, Wisoff JH, Snow RB, Souweidane MM, Bruce JN, Finlay JL. Delayed surgical resection of central nervous system germ cell tumors. Neurosurgery, 2002 50: 727-33; discussion 733-4.
[14] Hoffman HJ, Otsubo H, Hendrick EB, Humphreys RP, Drake JM, Becker LE, Greenberg M, Jenkin D. Intracranial germcell tumors in children. J Neurosurg, 1991 74: 545-551.

[15] Bouffet E, Baranzelli MC, Patte C, Portas M, Edan C, Chastagner $\mathrm{P}$, et al: Combined treatment modality for intracranial germinomas: results of a multicentre SFOP experience. Société Française d'Oncologie Pédiatrique. Br J Cancer 79:1199-204, 1999.

[16] Jennings MT, Gelman R, Hochberg F: Intracranial germ-cell tumors: natural history and pathogenesis. J Neurosurg 63: $155-167,1985$

[17] Noudel R, Vinchon M, Dhellemmes P, Litré CF, Rousseaux P. Intracranial teratomas in children: the role and timing of surgical removal. J Neurosurg Pediatr, 2008 2: 331-338.

[18] Packer RJ, Cohen BH, Cooney K. Intracranial germ cell tumors. The Oncologist 2000; 5: 312-320.

[19] Al-Tamimi YZ, Bhargava D, Surash S, Ramirez RE, Novegno F, Crimmins DW, Tyagi AK, Chumas PD. Endoscopic biopsy during third ventriculostomy in paediatric pineal region tumours. Child's Nerv Syst, 2008 24: 1323-1326.

[20] Chibbaro S, Rocco F Di, Makiese O, Reiss A, Poczos P, Mirone G, Servadei F, George B, Crafa P, Polivka M, Romano A. Neuroendoscopic management of posterior third ventricle and pineal region tumors: Technique, limitation, and possible complication avoidance. Neurosurg Rev, 2012 35: 331-338.

[21] Morgenstern PF, Osbun N, Schwartz TH, Greenfield JP, Tsiouris AJ, Souweidane MM. Pineal region tumors: an optimal approach for simultaneous endoscopic third ventriculostomy and biopsy. Neurosurg Focus, 2011 30: E3.

[22] O'Callaghan AM, Katapodis O, Ellison DW et al. The growing teratoma syndrome in a nongerminomatous germ cell tumor of the pineal gland: A case report and review. Cancer 1997; 80: 942-947.

[23] Jooma R, Kendall BE. Diagnosis and management of pineal tumors. J Neurosurg 1983; 58: 654-665.

[24] Ray P, Jallo GI, Kim RY et al. Endoscopic third ventriculostomy for tumor related hydrocephalus in a pediatric population. Neurosurg Focus 2005; 19: E8.

[25] O'Brien DF, Hayhurst C, Pizer B, Mallucci CL. Outcomes in patients undergoing single-trajectory endoscopic third ventriculostomy and endoscopic biopsy for midline tumors presenting with obstructive hydrocephalus. J Neurosurg Pediatr, 2008 105: 219-226.

[26] Wong T-T, Chen H-H, Liang M-L, Yen Y-S, Chang F-C. Neuroendoscopy in the management of pineal tumors. Child's Nerv Syst, 2011 27: 949-959.

[27] Grossman R, Sadetzki S, Spiegelmann R, Ram Z. Haemorrhagic complications and the incidence of asymptomatic bleeding associated with stereotactic brain biopsies. Acta Neurochir (Wien), 2005 147: 627-631.

[28] Lefranc M, Touzet G, Caron S, Maurage CA, Assaker R, Blond S. Are stereotactic sample biopsies still of value in the modern management of pineal region tumours? Lessons from a single-department, retrospective series. Acta Neurochir (Wien), 2011 153: 1111-1121. 
[29] Malone H, Yang J, Hershman DL, Wright JD, Bruce JN, Neugut AI. Complications Following Stereotactic Needle Biopsy of Intracranial Tumors. World Neurosurg, 2015 84: 1084-1089.

[30] Bamberg M, Kortmann R-D, Calaminus G, Becker G, Meisner C, Harms D, Göbel U. Radiation Therapy for Intracranial Germinoma: Results of the German Cooperative Prospective Trials MAKEI 83/86/89. J Clin Oncol, 1999 17: 2585-2585.

[31] Sawamura Y, Ikeda J, Shirato H et al. Germ cell tumours of the central nervous system: Treatment consideration based on 111 cases and their long-term clinical outcomes. Eur J Cancer 1998; 34: 104-110.
[32] Robertson PL, DaRosso RC, Allen JC. Improved prognosis of intracranial non-germinoma germ cell tumors with multimodality therapy. J Neurooncol 1997; 32: 71- 80.

[33] Calaminus G, Bamberg M, Jo“rgens H et al. Impact of surgery, chemotherapy and irradiation on long term outcome of intracranial malignant nongerminomatous germ cell tumors: Results of the German Cooperative Trial MAKEI 89. Klin Padiatr 2004; 216: 141-149.

[34] Calaminus G, Bamberg M, Harms D et al. AFP/beta-HCG secreting CNS germ cell tumors: Long-term outcome with respect to initial symptoms and primary tumor resection. Results of the cooperative trial MAKEI 89. Neuropediatrics 2005; 36: 71-77. 